\title{
Hollow Prosthetic Vaginal Dilator - A Novel Technique
}

\author{
Kasim Mohamed ${ }^{1}$, Preetha Krishnamurthy², Yogitha K³ , Syed Altaf Hussain ${ }^{4}$ \\ 1,2,3 Department of Prosthodontics, Crown \& Bridge, Sri Ramachandra Institute of Higher Education and \\ Research, Chennai, Tamilnadu, India. ${ }^{4}$ Department of Plastic Surgery, Cleft \& Craniofacial Centre, Sri \\ Ramachandra Institute of Higher Education and Research, Chennai, Tamilnadu, India.
}

\section{INTRODUCTION}

Certain rare congenital defects can occur during the prenatal development of the female reproductive system, resulting in an abnormal or absent vagina. ${ }^{1}$ It is a part of the female genital tract. It is muscular and an elastic organ with a soft epithelial lining making it susceptible to disruption during crucial times of organogenesis. ${ }^{1,2}$ There are different malformations that occur in the female genital organ, many of which are classified under the broader term "Müllerian duct anomalies".1,2 Müllerian agenesis is also referred to as congenital absence of the uterus and vagina (CAUV), müllerian aplasia (MA), genital renal ear syndrome (GRES), and Mayer-Rokitansky-KüsterHauser syndrome (MRKH). It has been considered as a sporadic anomaly, but the increase in familial cases now supports the hypothesis of a genetic cause characterised by a failure of the Müllerian ducts to develop, resulting in a missing uterus and variable malformations of the vagina. ${ }^{1-7}$ According to scientific data, its incidence is reported to be 1 in 4,000 to 10,000 females occurring due to a heterozygous transformation of WNT4 gene on chromosome 1p36.1-7 Additionally, traumatic vaginal injury in females, which is not uncommon, results in vaginal stenosis. ${ }^{5}$ This results in formation of scar tissue causing stricture of the vagina. ${ }^{5}$

Based on the family history, the patient must undergo medical examination supported by gynaecologic assistance to provide a sensitive and specific means of diagnosis. ${ }^{1,2}$ It is essential for the patients and their families to attend counselling before proceeding for treatment as this syndrome can cause severe psychological stress in a young woman. Patients with vaginal agenesis in MRKH syndrome can be treated by both surgical and non-surgical procedures and there has been no evidence of the superiority of one technique over another. ${ }^{6,11}$ Abbe-McIndoe operation is a common surgical method using split thickness skin graft for reconstruction and Frank's dilators is a common nonsurgical method for the creation of neovagina.3,7 Frank's initial technique used hand-held vaginal dilators in the lithotomy position and had technical limitations such as uncomfortable positioning, loss of time spent during the process of dilation, and fatigue of patient's hands. Ingram's method overcame these difficulties by using the sitting position to take advantage of the patient's body weight. 8,9 This method used vaginal dilators mounted on a specially designed bicycle seat stool that provided perineal pressure via the narrow and elevated anterior portion of the bicycle seat. ${ }^{10}$ Despite the favourable clinical outcome of this method, there were some practical difficulties in making and handling these in an outpatient clinic. However, irrespective of the procedure opted, prosthetic vaginal dilators or long-term vaginal stents are required to prevent the possible contraction of the reconstructed neovagina, to maintain vaginal width, depth and to avoid vaginal stenosis. ${ }^{6}$

\author{
Corresponding Author: \\ Dr. Preetha Krishnamurthy, \\ Department of Prosthodontics, \\ Crown \& Bridge, Sri Ramachandra \\ Institute of Higher Education and \\ Research, Porur, \\ Chennai-600102, \\ Tamilnadu, India. \\ E-mail: preethakrishnamurthy@gmail.com
}

DOI: $10.14260 / j e m d s / 2021 / 180$

How to Cite This Article:

Mohamed K, Krishnamurthy P, Yogitha K, et al. Hollow prosthetic vaginal dilator - a novel technique. J Evolution Med Dent Sci 2021;10(11):841-844, DOI: 10.14260/jemds/2021/180

Submission 04-11-2020,

Peer Review 20-01-2021,

Acceptance 27-01-2021,

Published 15-03-2021.

Copyright (c) 2021 Kasim Mohamed et al. This is an open access article distributed under Creative Commons Attribution License [Attribution 4.0 International (CC BY 4.0)] 
Most recently, there has been a trend toward first-line dilation therapy as it is patient centred and superior to surgery in terms of risk-benefit balance. ${ }^{11}$ Different materials such as acrylics and silicone-based materials have been used for its fabrication. Prosthetic vaginal dilators made with acrylic material are heavier than those made with silicone materials. These rigid or semirigid dilators tend to cause development of several complications, such as graft loss, pressure-related bladder or rectum perforations, fibrosis, contracture formation and most importantly, discomfort to the patients. Silicone materials have less complications and were found to be more comfortable for patients. However, the rough surface contributes to adherence of debris and difficulty in maintaining hygiene. As acrylic materials are harder than silicone materials, they tend to be more effective in achieving and maintaining the recommended size of the vagina. ${ }^{12}$ These vaginal dilators require precision in fabrication and in addition to the medical specialists, maxillofacial prosthodontists play a significant role in their fabrication as they are experts with the techniques and materials used. Thus, in order to provide the most comprehensive care to patients with vaginal agenesis, the treatment sequences have to be synchronised with medical and dental specialists. ${ }^{6}$

Considering the good response reported in previous studies with the use of acrylic dilators in women with vaginal stenosis, the medical team and patient opted for creation of a neovagina through the daily use of acrylic vaginal dilators. This article reports a case in which vaginal agenesis was successfully treated using a modified design of the conventional solid acrylic vaginal dilator.

\section{PRESENTATION OF CASE}

A 22-year-old woman diagnosed with MRKH syndrome karyotype 46, XX was referred to the Department of Plastic and Reconstructive Surgery by her gynaecologist for treatment of vaginal agenesis after she presented with primary amenorrhea and normal secondary sex characteristics. She had no significant medical history. Her general physical examination was normal with no features of associated skeletal abnormalities. Her breasts and pubic hair were Tanner stage IV. She had adequately oestrogenised external genitalia and a vaginal dimple of $0.5 \mathrm{~cm}$ in depth on external genitalia examination. However, uterus and cervix were not palpable on the recto-abdominal examination. Lab analysis revealed normal serum prolactin, thyroid stimulating hormone (TSH), follicular stimulating hormone (FSH) and luteinizing hormone (LH). Pelvic magnetic resonance imaging (MRI) confirmed a diagnosis of Müllerian agenesis with complete agenesis of the vagina. The ovaries were normal bilaterally with small rudimentary uterus. Urinary and rectal tracts were found to be normal.

The patient was explained about the surgical and the nonsurgical methods of management. After a thorough discussion of therapy, the patient felt highly motivated to use the non-operative procedure (vaginal dilators) due to the low morbidity, nonsurgical scarring and a near normal vaginal canal which could be created. The patient was then referred to the Department of Prosthodontics for the planning, formulation and serial moulding of the acrylic vaginal dilators that the patient was going to use for her treatment.

\section{DISCUSSION OF MANAGEMENT}

The patient was initially planned for treatment using a solid acrylic vaginal dilator for serial dilatation at the vaginal dimple. An arbitrary method of fabrication using rough measurement of the length and width was suggested by the gynecologist. ${ }^{2}$ Based on these measurements, a wax pattern (Hindustan Modelling Wax, India) was fabricated by the maxillofacial prosthodontist. The pattern was cylindrical, narrowing on the top to allow ease in insertion and a base to help in the removal. It was flasked, dewaxed, and processed in acrylic resin ((Dental Products of India (DPI), heat cure). The fabricated prosthesis was trimmed, finished and highly polished to achieve an smooth surface (Figure 1). The patient was advised to use the prosthesis with the help of a lubricant $\mathrm{K}-\mathrm{Y}$ jelly to allow easy and less painful insertion for a duration of 4 - 5 hours a day for a period of one month. Retention and pressure had to be applied and maintained by the use of a tight underwear. She was also instructed to maintain good personal hygiene and to maintain the stent clean. A thorough wash under fast running water and cleansing with a mild detergent was advised before and after usage of the vaginal dilator to prevent fungal growth. However, the patient was noncompliant due to initial bleeding at the site and the sheer weight of the mould which she was unable to hold at the site.

The patient reported a month later and was advised to continue the treatment process. To overcome the difficulties, certain modifications were incorporated in the conventional design of the vaginal dilator. A hollow wax pattern measuring $5 \mathrm{~cm}$ in length and $2 \mathrm{~cm}$ in outer diameter with a small conical tip and a flat base was fabricated using modelling wax (Hindustan Modelling Wax, India). 2 window cuts measuring 5 x $5 \mathrm{~mm} \mathrm{sq}$ area was made, one near the flat base and the other near the conical tip (Figure 2). During flasking, the hollow portion was filled with orthocal (type $\mathrm{V}$ gypsum) and was placed in the mixture of dental plaster (type I gypsum). The 2 window cuts helped in positioning the wax pattern and allowed the orthocal portion to join the plaster component, enabling complete elimination of the wax (Figure 3). Processing was then done with heat cure acrylic resin. Following processing, through the window cuts, the orthocal was removed and the hollowness was maintained. The window cuts were then sealed using self-cure resin. The processed hollow acrylic vaginal dilator was then trimmed, finished and polished to achieve a highly smooth surface (Figure 4). After the patient was given instructions regarding the use, she began using the dilator, she was followed up every 1 week for measuring the vaginal length. She was instructed to use the dilators by increasing the length and width progressively until a satisfactory vaginal orifice was created. On reviewing after a period of one month the patient was found to be using the dilator with comfort. A dilator of $6 \mathrm{~cm}$ length and $2.3 \mathrm{~cm}$ diameter was introduced and after 4 weeks of treatment, the vaginal dilator was increased to a dimension of $8 \mathrm{~cm}$ in length and $2.5 \mathrm{~cm}$ in diameter. Holes measuring 0.8 $\mathrm{mm}$ were made on the superior conical surface to facilitate draining of the discharge (Figure 5). The patient was asked to clean the vaginal dilator everyday using a mild detergent under running tap water and to use a denture cleanser like Cleansodent weekly. The treatment took place without significant complications and there was no dilation of the urethral meatus or any vaginal prolapse. 

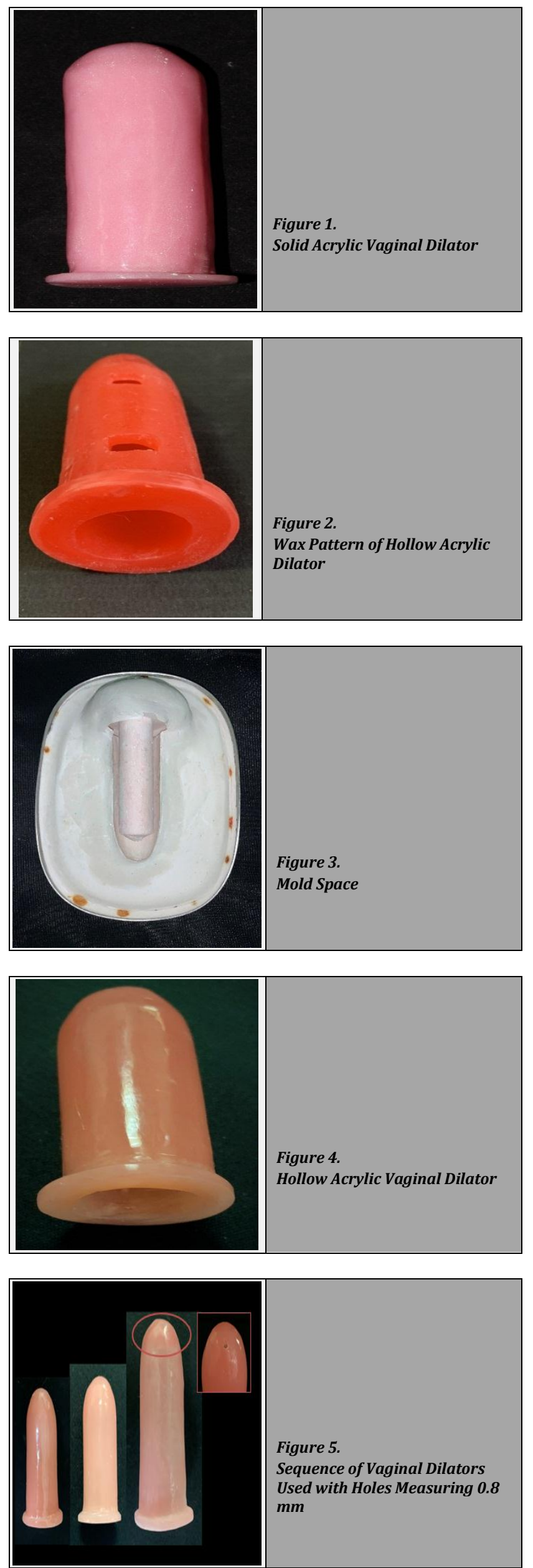

\section{DISCUSSION}

Mullerian agenesis is a rare congenital sporadic anomaly that affects the female reproductive system. ${ }^{2}$ There are various surgical methods for creation of neovagina, however, the most common complication after surgical reconstruction of vaginal is scar tissue formation leading to restenosis, necessitating persistent perineal dilatation using vaginal dilators (VD) after surgery to maintain the caliber of the neovagina. This can lead to noncompliance or to significant complications including vaginal bleeding, perforation of the vagina or bladder, fistula formation and secondary vaginal surgery after vaginoplasty which can have profound and long-lasting effects on young girls and women. Unlike the normal vagina which is a selfcleansing organ, epithelial debris and sebaceous secretions tend to accumulate in the skin lining the neovagina making it difficult to maintain it clean and odour free. ${ }^{13,14}$ These factors can have effect on the patient's quality of life. So, surgical approaches have been increasingly replaced with conservative techniques with fewer complications. ${ }^{2}$

Methods using dilators are considered as the first-line therapy because they have minimal morbidity and complications, low cost, total patient control, high success rate (75 - $85 \%$ ), natural self-cleansable lining, and they allow outpatient follow-up without requiring hospitalisation. The classic method of non-surgical management involves the use of rigid acrylic dilators as advocated by Frank in 1938 and Ingram's modified technique. ${ }^{15}$ These vaginal dilators are smooth, cylindrical devices that are inserted into a woman's vagina to facilitate stretching and relaxation of the underlying tissues. ${ }^{16}$ Due to its elastic nature, the vaginal tissue has the tendency to expand during the insertion of a dilator. ${ }^{6}$ To accomplish the tissue expansion, customised dilators are made with increase in size over a period of time. ${ }^{6}$ They progress from one designated size (length and diameter) to another in a stepwise fashion and are indicated only when the vaginal dimple is deep enough $(2-4 \mathrm{~cm}) .6,16$ Sangeetha et al., suggested that the predetermined dimensions of the prosthetic devices aid in the successful neovaginal reconstruction. Similarly in the present case, the prosthetic device was fabricated according to the dimensions suggested by the gynaecologist.12 Although the use of the conventional solid rigid acrylic dilators have proved to be successful, they may cause complications such as urethritis, cystitis, vesical or rectovaginal fistula, secondary genital prolapse, and even vaginal necrosis. These complications are commonly witnessed when the dilators are not used sequentially and recall and reviews have not been performed. A lack of compliance to wear a dilator, even if it is inconvenient, is known to be the main cause of failure. ${ }^{6}$ A common reason stated by patients is pain and discomfort from solid acrylic dilator therapy.

To overcome these difficulties, we modified the conventional solid acrylic vaginal dilator using a unique fabrication technique into a hollow acrylic dilator. This hollow dilator reduced the weight of the prosthesis and weighed only one-third of the solid dilator. ${ }^{2}$ Patient reported ease and comfort while using the hollow dilator as it was easy to insert and remove, thus reducing the chances of complications faced earlier with solid acrylic dilator. The hollow component also aided in draining discharge when present or when the dilator reached the cervix. ${ }^{2}$ Thus, a hollow dilator with the same 
dimensions as that of a solid dilator could bring about better results and reduce the complications. An ideal dilation treatment duration includes a long-term commitment of over 3 - 6 months. This information may help patients set realistic goals and expectations for their treatment as poorly defined intervention regimes shifting from one size to another is cumbersome and confusing. ${ }^{16}$ Patients also require regular counselling and motivation during therapy. Though urethral dilation has been considered a serious risk while using vaginal dilators, the complication rate after surgery was greater, with high morbidity as half of patients needed secondary procedures. ${ }^{11}$ Additionally, the dilators are cost-effectiveness than surgery. ${ }^{11}$ Nevertheless, the procedure of fabrication requires considerable skill and is technique sensitive thus posing a challenge even to a maxillofacial prosthodontist with proficient knowledge in the design and fabrication of vaginal dilators.

\section{CONCLUSIONS}

Treatment of vaginal aplasia in MRKH syndrome with the goal of creating a neovagina can be performed by surgery or with the use of dilators. Both methods take approximately 6 months to obtain the maximum result. The use of inexpensive and easily accessible acrylic vaginal dilators may be an effective and non-invasive alternative with few side effects for women with vaginal agenesis, particularly in the developing countries. Despite the good results of dilation therapy reported since the 1930s, surgeons are still looking for the best surgical technique. ${ }^{11}$ Efforts should be made to improve results with the development of validated dilation programs and therapeutic education by multidisciplinary experienced teams, in association with the patients. ${ }^{11}$

Financial or other competing interests: None.

Disclosure forms provided by the authors are available with the full text of this article at jemds.com.

I would like to personally thank Dr. G. Usha Rani, Department of Obstetrics and Gynaecology, Sri Ramachandra University, Porur, Chennai, India for her untiring support.

Authors acknowledge Dr. Deyonna, Department of Plastic and Reconstructive Surgery, Sri Ramachandra University, Porur, Chennai, India.

\section{REFERENCES}

[1] Rasmi P, Padmanabhan T, Mohammed K, et al. Prosthetic vaginal dilator - a case report. The Internet Journal of Gynecology and Obstetrics 2010;15(1).
[2] Gnanavi P, Kumar KR, Gomathi G, et al. Hollow vaginal dilator - a case report. IOSR Journal of Dental and Medical Sciences (IOSR - JDMS) 2017;16(12):09-13.

[3] Rathee M, Singhal SR, Malik S, et al. Resilient customized hollow vaginal stent for the treatment of vaginal agenesis in Mayer-Rokitansky-Kuster-hauser syndrome. Niger J Surg 2020;26(1):88-91.

[4] Singh G, Singh R, Gambhir RS, et al. Fabrication of a prosthesis for a patient with vaginal agenesis. J Fam Med Community Health 2016;3(5):1093.

[5] Dutt P, Chand P, Jurel SK, et al. Customized prosthetic vaginal dilator in genital trauma. Indian Dental Journal 2016;8:60-4.

[6] Kamalakannan J, Murthy V, Kularashmi BS, et al. Customized silicone vaginal stent. J Obstet Gynecol India 2015;65(4):281-3.

[7] Rathee M, Boora P, Kundu R. Custom fabricated acrylic vaginal stent as an adjunct to surgical creation of neovagina for a young female with isolated vaginal agenesis. J Hum Reprod Sci 2014;7(4):272-5.

[8] Frank RT. The formation of an artificial vagina without operation. American Journal of Obstetrics and Gynecology 1938;35(6):1053-5.

[9] Ingram JM. The bicycle seat stool in the treatment of vaginal agenesis and stenosis: a preliminary report. Am J Obstet Gynecol 1981;140(8):867-73.

[10] Roberts CP, Haber MJ, Rock JA. Vaginal creation for müllerian agenesis. Am J Obstet Gynecol 2001;185(6):1349-53.

[11] Cheikhelard A, Bidet M, Baptiste A, et al. Surgery is not superior to dilation for the management of vaginal agenesis in Mayer-Rokitansky-Küster-Hauser syndrome: a multicenter comparative observational study in 131 patients. Obstetrical \& Gynecological Survey 2019;74(2):79-80.

[12] Patnana A, Chugh A, Chugh V, et al. Simple and novel technique for fabrication of prosthetic vaginal dilators. BMJ Case Rep 2019;12(4):e229524.

[13] Selvaggi G, Monstrey S, Depypere H, et al. Creation of a neovagina with use of a pudendal thigh fasciocutaneous flap and restoration of uterovaginal continuity. Fertil Steril 2003;80(3):607-11.

[14] Fedele L, Bianchi S, Berlanda N, et al. Neovaginal mucosa after Vecchietti's laparoscopic operation for Rokitansky syndrome: Structural and ultrastructural study. Am J Obstet Gynecol 2006;195(1):56-61.

[15] Ogliari KCM, Da Silva Rios S, Chen ACR, et al. An alternative approach to vaginal dilation in patients with Meyer-Rokitanski-Küster-Hauser syndrome: two case reports. Gynecol Endocrinol 2020;36(8):746-8.

[16] Liu M, Juravic M, Mazza G, et al. Vaginal dilators: issues and answers. Sex Med Rev 2020;S2050-0521(19)301258. 